\title{
Using an IBM PC to network Walter/Palya experiment controllers
}

\author{
MARK E. PEVEY \\ Emory University, Atlanta, Georgia
}

\begin{abstract}
A communications module that provides for the convenient use of the Walter/Palya experiment controller with an IBM PC is discussed. Following the user's responses to prompts for apparatus and experimental procedure, the module appropriately loads the controller. It also automatically uploads and stores experimental data. Error checking is transparent to the user. Personal gettingstarted experiences with the experiment controller are also discussed.
\end{abstract}

This paper provides information concerning the implementation of the Walter/Palya controller on an IBM PC. Primarily, I describe the software utility that I use to communicate with the experiment controller. Secondarily, I share some of my experiences getting started.

The Walter/Palya experiment controller is a completely self-contained printed circuit board computer interface. It has a microcomputer that runs an experiment and all the interface hardware necessary to connect directly to the experimental apparatus and directly to an IBM PC, $\mathrm{XT}, \mathrm{AT}$, or PS/2. Experiment control commands are written in a specially written BASIC (ECBASIC), which contains high-level instructions for every aspect of experiment control. No assembly language calls are necessary, and no awkward syntax is used. ECBASIC "feels" the same as BASIC itself. ECBASIC is in firmware; as a result, when power is applied to the controller, ECBASIC comes up running.

The editor under MS-DOS on the IBM PC is used to write programs following the conventions of ECBASIC. All the functionality of MS-DOS is available for this task. Programs are stored on the disk and, in fact, can be cut and pasted together to create programs containing previously used elements. In this sense, BASIC can have the same macro functionality as FORTH. Programs are sent to and received from the controller in the same way that communications between the computer and a modem or printer take place.

Although commercially available modem communications programs can provide all the functionality necessary to run experiments with the Walter/Palya controller, I use RBAS, a public domain program. ${ }^{1}$ It has a variety of useful functions.

After programs are written in ECBASIC with an editor or word processor and are stored on the disk, then RBAS can be run in the same manner as any other MSDOS program. RBAS asks which apparatuses are to be

The author gratefully acknowledges W. L. Palya's willingness to put his boards into the public domain and to accept phone calls when technical questions arose. Requests for reprints should be sent to the author, Department of Psychology, Emory University, Atlanta, GA 30322. used, what experiments are to be run using each, and what file names are to be used for the resulting data. It automatically loads the chambers, starts the boxes running, collects the data, and stores the data on the disk when the experiments are over. If, while the experiments are running, the PC is needed for any other task, RBAS can be terminated. Because the experiments are run as independent tasks in peripheral processors, they are unaffected by the PC. (In fact, the PC can be turned off.) An advantage of the network concept is that $100 \%$ of the resources of the $\mathrm{PC}$ are available for other uses while experiments are running. When RBAS is started again, it resumes its monitoring and data collection functions.

\section{INVOKING RBAS}

RBAS is invoked by typing the following:

$$
\text { RBAS <cr }>
$$

Alternatively, RBAS can be invoked with two optional parameters: port number and baud rate. In this case, RBAS is invoked by typing the following:

$$
\text { RBAS [port] [baud] }
$$

Port number is set to either 1 (when the experiment controllers are connected to COM1) or 2 (when the controllers are connected to COM2). Baud rate (the transmission speed) is set to match the baud rate at which the controllers have been set. RBAS defaults to COM1 at 9600 baud if no arguments are provided. For any other setting, both calling parameters must be provided.

If any illegal calling parameters are specified, the following message will be printed.

\section{USAGE: RBAS [PORT] [BAUD] \\ PORT NUMBERS ARE 1 FOR COM1, 2 FOR COM2 VALID BAUD RATES ARE $9600,4800,2400,1200$, 600, AND 300 \\ NO ARGUMENTS DEFAULTS TO COM1 AND 9600 BAUD}

For an IBM PC or an IBM PC/XT, communications to the experiment controllers should be at 4800 baud. The 
XT will communicate at 9600 baud, but there will be occasional errors. If errors are experienced in uploading or downloading information, the XT or PC may be having trouble keeping up. Set the controller and the RBAS calling parameter to a slower baud rate.

When RBAS starts, it prompts the user with the following:

\section{COMMAND:}

The following responses are those recognized by RBAS (all are single-character commands):

\section{S-START (download and start experiment) \\ R-RESTART (get data from ongoing experiments) \\ K-KILL (terminate an experiment) \\ D-DISPLAY (display status of experiments) \\ C-CONNECT (act as a terminal on a controller) \\ Q-QUIT (terminate RBAS and close files)}

A "Control-Break" aborts any of these routines. RBAS then returns to the Command prompt.

\section{Start}

To start an experiment, " $S$ " is typed following the Command prompt. RBAS then prompts the user with:

\section{ENTER BOX NUMBER:}

An experimental apparatus number from 1 to 9 is then entered. If the apparatus number entered is one that is already running, RBAS prints the following message:

\section{BOX XX IS ACTIVE}

Assuming there is no error at "Box Number," RBAS then prompts for the name of the ECBASIC program that implements the experimental procedures for the current session in that apparatus:

\section{ENTER PROGRAM NAME:}

After the prompt, the name of the file is entered, followed by a carriage return. The file need not be in the default directory. If it is in some other subdirectory, the appropriate subdirectory name and the file name in normal MSDOS format are entered. If the file specified is not found, RBAS responds with:

\section{CANNOT FIND < FILE >}

Assuming there is no error at "Program Name," RBAS prompts for the name of the file in which that day's data for that experimental apparatus are to be stored:

\section{ENTER DATA FILE NAME:}

The user then enters the name of the data file, followed by a carriage return. Again, this file can be created in any subdirectory by using normal MS-DOS format. When the file name is entered, RBAS checks to see if the data file already exists. If it does, RBAS responds with:

\section{FILE EXISTS, OVERWRITE IT? (Y OR N)?}

If the response is "Y," RBAS overwrites the file. If the response is " $\mathrm{N}$," RBAS reprompts for a new data file name. If the specified file cannot be created, RBAS prints the message

\section{CANNOT CREATE < FILE >}

and reprompts for the data file name.

Before RBAS starts an experimental session, it creates and opens the specified data file, sends the program to the specified controller, and then tries to start it. If there are no problems, RBAS returns with the command prompt. However, if the controller does not respond properly, RBAS writes the command that caused the error as well as the message sent from the controller into the data file. The load is aborted, and RBAS prints the message

\section{NO RESPONSE FROM BOX XX}

If this error occurs, the apparatus is probably turned off, is in diagnostic mode, or is disconnected. Correct the fault, reset the controller, and try again.

As previously mentioned, when RBAS is running, it constantly checks the network for experiments that have been completed. When a controller is finished running an experiment and is therefore ready to upload its data, it waits for a go-ahead signal from RBAS. (For a more detailed discussion of network protocols and functions, refer to the ECBASIC networking chapter (p. 59) of the Document Set for Experiment Controller by Walter \& Palya, 1985.) ${ }^{2}$ When RBAS gives a controller permission to upload, the experiment controller obliges and RBAS collects and stores the data in the specified data file and then closes the file. Note that ECBASIC programs should output data at noncritical times since it could take several seconds for RBAS to poll the unit and upload the data. The controller halts at print statements until the data is transferred. Normally, print statements are placed at the end of the program after the experiment is over. If a PRINT "*DONE*" statement follows the data, then RBAS signifies that that experiment has completed by printing on the screen:

\section{BOX XX COMPLETE}

IF RBAS is exited while experiments are running, all open data files are closed. The controllers, nevertheless, continue to run and collect data as if nothing had changed. When the controller is ready to upload, it waits for the go-ahead signal from RBAS, as always. If RBAS is not running, the controllers continue to wait for the authorization to upload indefinitely with no data loss. If RBAS is restarted, it can again monitor the network, reopen all data files, and collect data from any controllers ready to upload.

\section{Restarting RBAS}

RBAS does not store a record of the experiments that were active when it was exited. Consequently, if RBAS is restarted, the user must provide RBAS with the names of the experiments that had been running. RBAS is 
restarted by invoking RBAS and then entering " $R$ " in response to the command prompt.

The restart procedure is similar to the start-up procedure. RBAS prompts with

\section{ENTER BOX NUMBER:}

The number of an apparatus that was running before RBAS was exited is then entered. Next, RBAS prompts for the data file name:

\section{ENTER DATA FILE NAME:}

If the file name specified already exists, RBAS prompts with

\section{FILE EXISTS, OVERWRITE, APPEND, OR REENTER? (O, A, or $\mathrm{R})$ ?}

If an $O$ is specified, the file is overwritten. If an $R$ is specified, the program reprompts for the data file name. If an $A$ is specified, data is written, starting at the end of the file specified. If the file specified cannot be created, the message

\section{CANNOT CREATE < FILE $>$}

is printed, and RBAS reprompts for the data file name. RBAS then returns to the command prompt and begins collecting data. This restart procedure is repeated for each experiment that was running when RBAS was exited.

\section{Kill}

The Kill command is used to immediately terminate an experiment. Again, RBAS prompts for a specific experimental apparatus number:

\section{ENTER BOX NUMBER:}

The program for the specified controller is halted and the data file is closed. RBAS then returns with the Command prompt.

\section{Display}

The Display command indicates the status of the current experiments. The Display command is executed by typing " $D$ '" at the command prompt. RBAS lists all active experiments in the following manner:

\section{BOX 1 ACTIVE \\ BOX 2 ACTIVE}

If all experiments are completed, RBAS prints the following message:

\section{NO UNITS ARE ACTIVE}

This message also occurs if RBAS has just been restarted. RBAS initially assumes that no controllers are running until it is told which experiments had been active when RBAS had been terminated.

\section{Connect}

The Connect command allows the user to establish a dedicated link directly to a particular controller. The PC then acts as a terminal, allowing the user to communicate interactively with a specific experiment controller. This is equivalent to having a terminal connected directly to a single controller in stand-alone mode (see the standalone mode section, p. 122, Walter \& Palya, 1985). This mode is very useful in debugging ECBASIC programs. For example, if you type TURNON 4, light number 4 goes on. If you type PRINT A, the value of variable $A$ is printed. It is important to note, however, that RBAS cannot function as a network communication controller for other boxes while in the direct connect mode.

The connect mode is entered by typing $\mathrm{C}$ at the command prompt. RBAS prompts for the number of the apparatus to which the user wishes to connect:

\section{ENTER BOX NUMBER:}

When the controller number has been entered, RBAS prints the following:

\section{TYPE CONTROL-BREAK TO EXIT LINK OK}

When the "OK" prompt appears, the direct link has been established. "OK" is the ECBASIC prompt sent from the experiment controller itself. The experiment controller is then an independently functioning computer waiting for input from the keyboard. At this point, ECBASIC commands can be sent directly to the controller and the responses will print directly to the screen. To break the link, type "Control-Break." This returns to the command mode.

\section{Quit}

The Quit command exits RBAS and returns to MSDOS. When RBAS is exited, the experiments continue to run undisturbed. However, the data generated by the experiments are not automatically stored on the disk. RBAS must be restarted to obtain that functionality. When RBAS is exited, all open files are closed. To reopen the files for data collection, RBAS must be reinstituted through the Restart command.

\section{PERSONAL EXPERIENCES IN SETTING UP A COMPUTERIZED LAB}

I have been using the Walter/Palya experiment controllers for more than a year and am very pleased with the results. These controllers are inexpensive, easy to build, easy to maintain, and easy to use. The idea of building a computer initially was very frightening; however, I built my first controllers with no experience whatsoever in soldering or electronics. Eight boards were needed, I soldered 12 boards. Ten worked the first time they were 
plugged in. Two had to be discarded. The boards are so inexpensive that they are best seen as expendables rather than as equipment. In retrospect, building the controllers was about the same as building a Heath Kit project. All that was required to build the controllers was diligence and meticulousness. The experiment controller manual is exceptionally informative and well written. It predicted and answered virtually every question that arose.

I have now been involved in all phases of the installation of controllers at two sites. In the first laboratory, each experimental station was set up as an isolated unit. In the second, the controllers were added on to an already existing system, and an attempt was made to leave as much of the existing relay rack wiring as possible. These two approaches resulted in dramatically different outcomes.

At the first laboratory, the network was made of simple elements, because the controllers and power supplies were mounted on the chambers themselves. Getting the network up and running was relatively quick and painless. At the second laboratory, on the other hand, trying to layer controllers into an already existing system of patched and repatched cables produced innumerable problems, all of which were eventually traced to undocumented or faulty connectors in the old setup, or to a common power supply running all experimental stations.
I therefore strongly recommend constructing a network of completely isolated, separate, identical units. Simply attach the controller and power supply to the chamber. This allows for minimal cable length and makes a nice neat package. Cables should never be any longer than necessary. Long cables that go through several sets of connectors allow more places for problems to occur. In a network of isolated units, failures affect only one experimental station and not the entire network. Additionally, independent self-contained experimental stations and their resulting interchangeable parts make the job of debugging dramatically easier.

\section{REFERENCE}

Walter, D. E., \& Palya, W. L. (1985). Document set for experiment controller. Jacksonville, AL: Jacksonville State University, Department of Psychology.

\section{NOTES}

1. RBAS was written by D. E. Walter and is available from W. L. Palya, Department of Psychology, Jacksonville State University, Jackonsville, AL 36265.

2. This manual is available from W. L. Palya, Department of Psychology, Jacksonville State University, Jacksonville, AL 36265. 